Meta

Journal des traducteurs

Translators' Journal

\title{
L’interprétation en milieu judiciaire
}

\section{Gilles Bergeron}

Volume 47, numéro 2, juin 2002

Traduction et terminologie juridiques

URI : https://id.erudit.org/iderudit/008011ar

DOI : https://doi.org/10.7202/008011ar

Aller au sommaire du numéro

\section{Éditeur(s)}

Les Presses de l'Université de Montréal

\section{ISSN}

0026-0452 (imprimé)

1492-1421 (numérique)

Découvrir la revue

\section{Citer cet article}

Bergeron, G. (2002). L'interprétation en milieu judiciaire. Meta, 47(2), 225-232. https://doi.org/10.7202/008011ar

\section{Résumé de l'article}

Le droit au service d'un interprète dans le cadre de procédures de cour est consacré dans les Chartes des droits canadienne et québécoise et, par conséquent, constitue donc un élément important de l'administration de la justice au Canada. Les tribunaux ont interprété les dispositions de ces chartes et ont développé des normes d'interprétation auxquelles nous sommes confrontés et dont le respect n'est pas sans poser certains problèmes. Bien sûr, ce droit constitutionnel n'a pas le même impact d'une région à l'autre. La région de Montréal, par exemple, présente un portrait multiethnique particulier puisque environ $90 \%$ de la population immigrante du Québec y résident. Cette diversité culturelle qui caractérise la région vient donc nécessairement teinter les services judiciaires qui y sont offerts. La conjugaison évolutive de ces droits, de ces normes et de ces réalités socioculturelles se présente donc, pour une cour comme celle de Montréal, comme un défi constant à relever dans le cadre d'une saine administration de la justice. 


\title{
L'interprétation en milieu judiciaire
}

\author{
GILLES BERGERON \\ Avocat, greffier adjoint à la Cour municipale de Montréal, Montréal, Canada
}

\begin{abstract}
RÉSUMÉ
Le droit au service d'un interprète dans le cadre de procédures de cour est consacré dans les Chartes des droits canadienne et québécoise et, par conséquent, constitue donc un élément important de l'administration de la justice au Canada. Les tribunaux ont interprété les dispositions de ces chartes et ont développé des normes d'interprétation auxquelles nous sommes confrontés et dont le respect n'est pas sans poser certains problèmes. Bien sûr, ce droit constitutionnel n'a pas le même impact d'une région à l'autre. La région de Montréal, par exemple, présente un portrait multiethnique particulier puisque environ $90 \%$ de la population immigrante du Québec y résident. Cette diversité culturelle qui caractérise la région vient donc nécessairement teinter les services judiciaires qui y sont offerts. La conjugaison évolutive de ces droits, de ces normes et de ces réalités socioculturelles se présente donc, pour une cour comme celle de Montréal, comme un défi constant à relever dans le cadre d'une saine administration de la justice.
\end{abstract}

\begin{abstract}
The right to the services of an interpreter within the framework of court procedures is enshrined in both the Canadian and the Quebec Charter of Rights, and therefore, constitutes an important element in the administration of justice in Canada. The courts have interpreted the provisions of these Charters and have developed standards of interpretation with which we are faced and of which application is not without problems. Certainly, this constitutional right does not affect all regions in the same way. For example, the Montreal region has a multiethnic character all its own, as $90 \%$ of all immigrants who settle in Quebec live here. This cultural diversity, which characterizes the region, will necessarily affect the judicial services offered. The combined evolution of these rights, norms, and sociocultural realities therefore represent, for a court such as Montreal's, a constant challenge within the framework of a sound administration of justice.
\end{abstract}

\section{MOTS-CLÉS/KEYWORDS}

common law, fidélité de l'interprétation, multiculturalisme, interprétation au Canada

\section{L'interprétation judiciaire au Canada : perspective montréalaise}

L'interprétation au Canada, où la population est de plus en plus caractérisée par une mixité culturelle, est devenue un service important, incontournable, voire vital dans certaines circonstances et ce, dans plusieurs domaines tels qu'en matière médicale, sociale ou économique. Le monde judiciaire n'y fait pas exception. Bien que le droit à l'interprétation devant les tribunaux soit reconnu en common law depuis près d'un siècle, l'importance grandissante du multiculturalisme dans notre société a forcé un raffinement des principes afférents à ce droit ainsi qu'un ajustement nécessaire de l'appareil judiciaire.

L'objet du présent texte n'est pas de présenter une analyse juridique détaillée du droit aux services d'interprétation au Canada. Je me contenterai de résumer les 
grands principes qui ont émergé quant à l'exercice de ce droit, tant des pouvoirs législatifs que judiciaires, des normes d'interprétation définies par la Cour Suprême du Canada, les principaux problèmes qui se présentent dans l'exercice de ce droit et les solutions possibles qui peuvent être envisagées, notamment dans une perspective d'administration de la justice montréalaise.

C'est dans une décision anglaise, R. c. Lee Kun, [1916] 1 K.B. 337 (C.C.A.), que l'on retrouve les premiers grands principes émis à l'égard du droit à l'interprétation dans les procédures judiciaires. Lord Reading, qui rendait jugement pour la Court of Criminal Appeal, a émis les commentaires suivants, qui ont été adoptés par la suite comme principes en matière de droit à l'interprétation:

- un accusé qui n'est pas représenté, qui ne parle pas et ne comprend pas la langue du tribunal, a droit à ce que la preuve soit traduite dans sa langue;

- un accusé représenté a le même droit sauf s'il y a renoncé clairement et que le juge est convaincu qu'il comprend l'essence de la preuve qui est produite contre lui;

- la présence de l'accusé à son procès est nécessaire pour entendre la preuve présentée contre lui et pour pouvoir y répondre, cette présence ne signifiant pas seulement la présence physique mais également la faculté de comprendre la nature des procédures;

- les tribunaux ont une obligation positive de s'assurer que les personnes accusées et dont la liberté est menacée comprennent et soient comprises.

Ces principes ont donc alimenté les tribunaux pendant plusieurs années lorsqu'il était question de droit aux services d'interprètes.

En 1960, une loi fédérale, la Déclaration canadienne des droits, L.R.C. (1985), app. III, reconnaissait expressément le droit à l'assistance d'un interprète. L'alinéa $2 \mathrm{~g}$ ) de cette loi se lit ainsi:

2. ... nulle loi du Canada ne doit s'interpréter ni s'appliquer comme...

g) privant une personne du droit à l'assistance d'un interprète dans des procédures où elle est mise en cause ou est partie ou témoin, devant une cour, une commission, un office, un conseil ou un autre tribunal, si elle ne comprend ou ne parle pas la langue dans laquelle se déroulent les procédures.

En 1975, le gouvernement du Québec adoptait la Charte des droits et libertés de la personne, L.R.Q., ch. C-12, et dont l'article 36 se lit ainsi :

36. Tout accusé a le droit d'être assisté gratuitement d'un interprète s'il ne comprend pas la langue employée à l'audience ou s'il est atteint de surdité.

Enfin, en 1982, l'article 14 de la Charte canadienne des droits et libertés établissait que:

14. La partie ou le témoin qui ne peuvent suivre les procédures, soit parce qu'ils ne comprennent pas ou ne parlent pas la langue employée, soit parce qu'ils sont atteints de surdité, ont droit à l'assistance d'un interprète.

Il est intéressant de noter que le texte de la Déclaration canadienne des droits fait abstraction des personnes sourdes ce qui n'est pas le cas de la Charte québécoise qui limite cependant le droit à l'assistance d'un interprète aux «accusés», donc en matière criminelle seulement. Le texte québécois établit toutefois la gratuité des services d'interprétation, ce que les deux autres textes ne prévoient pas. Enfin, la Charte canadienne étend le droit en question au-delà des matières criminelles et protège également les personnes atteintes de surdité. Les règles de common law développées à partir de l'affaire Lee Kun sont heureusement venues pallier, de façon générale, les lacunes de ces textes de loi. 
Ce n'est que douze ans après l'entrée en vigueur de la Charte canadienne que la Cour Suprême du Canada, dans l'affaire R. c. Tran, [1994] 2 R.C.S. 95, a eu l'occasion d'interpréter son article 14 et d'en établir un cadre d'analyse. Cette décision unanime d'un banc de sept juges de la cour, constitue, encore aujourd'hui, la référence principale en ce qui concerne le droit à l'assistance d'un interprète dans le cadre de procédures judiciaires au Canada.

Retenons les principes suivants, réitérés ou dégagés par la Cour Suprême dans cette décision:

- Le droit d'un accusé d'être présent à son procès (codifié à l'article 650 du Code criminel), va au-delà de la simple présence physique. Cette présence signifie également que l'accusé doit être capable de comprendre la langue des procédures.

- Le droit prévu à l'article 14 est un aspect du droit à un procès ou une audition équitable qui englobe le droit d'un accusé d'être informé de la preuve produite contre lui et son droit à une défense pleine et entière. De plus, il est lié à d'autres droits et garanties juridiques prévus à la Charte, comme par exemple, le droit à un procès public, le droit à la communication de la preuve, les droits à l'égalité, les droits des autochtones ainsi que «la prétention d'être une société multiculturelle, exprimée en partie à l'article $27 »$.

- Les questions concernant une violation de l'article 14 de la Charte seront analysées selon un schéma en trois étapes:

- L'identification du besoin d'un interprète. Soulignons ici que les tribunaux devront être généreux et faire preuve de compréhension lorsqu'une demande d'interprète leur sera adressée. Un refus devra être justifié par une preuve solide et convaincante que la demande constitue un abus de procédure. Le juge n'est pas tenu d'informer systématiquement les accusés de ce droit ni de tenir une enquête pour s'assurer du respect de celui-ci. À l'inverse, l'accusé n'est pas tenu de faire valoir formellement son droit à l'assistance d'un interprète. Les tribunaux ayant le devoir d'assurer l'équité dans leurs procédures, ils doivent intervenir s'ils se rendent compte que ce droit n'est pas respecté.

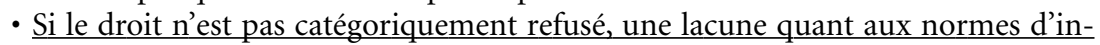
terprétation devra être démontrée. Ces normes correspondent à la continuité, la fidélité, l'impartialité, la compétence et la concomitance. Nous y reviendrons un peu plus loin.

- La lacune en question doit s'être produite dans le cadre de procédures où les intérêts vitaux de l'accusé étaient en jeu. En fait, ce droit s'applique pratiquement à toutes les étapes des procédures au cours desquelles l'affaire progresse; les témoignages, les argumentations des procureurs ou les directives du juge au jury par exemple. Les questions purement administratives telle que la consultation du calendrier judiciaire pour déterminer une date d'audition, sont toutefois exclues de l'application de ce droit.

- Compte tenu de l'importance du droit à l'interprète, aucun préjudice n'a à être prouvé. La simple violation de ce droit constitue un préjudice en soi.

- La réparation qui sera ordonnée dans le cas d'une violation du droit prévu à l'article 14, sera ordinairement d'annuler la déclaration de culpabilité et d'ordonner un nouveau procès ou une nouvelle audition concernant la sentence.

- La renonciation au droit à l'interprète sera, dans la majorité des cas, impossible à accorder compte tenu du caractère fondamental de ce droit. Autrement, la renonciation devra être faite personnellement et le tribunal devra s'assurer, par l'intermédiaire d'un interprète, que l'accusé réalise pleinement ce qu'il fait. Si un avocat représente l'accusé, il devra convaincre le tribunal que «la nature du droit et l'effet de la renonciation sur ce droit ont été expliqués à l'accusé» dans sa langue. 
Ajoutons à ces principes, développés dans une affaire criminelle, que le droit à l'assistance d'un interprète prévu à l'article 14 de la Charte, s'étend également aux procédures en matières administratives et civiles. La question des coûts relatifs à la prestation des services d'interprète dans les causes civiles, en demeure toutefois une d'intérêt. Le ministère public, dans de tels cas, semble, pour le moment, exempté de ces dépenses, les frais devant être assumés par l'une ou l'autre des parties en cause.

La norme d'interprétation au Canada établie par la Cour Suprême est donc composée de cinq éléments. Le premier est la continuité de l'interprétation. La Cour mentionne que le droit d'un accusé d'entendre et de comprendre les procédures est un droit continu. Les résumés de témoignage et les interruptions sont donc à proscrire. La majorité des plaintes formulées par les juges à la Cour municipale de Montréal en 1999 et 2000, concerne cette tendance de certains interprètes à résumer les discussions ou les témoignages.

Le deuxième élément concerne la fidélité de l'interprétation. La Cour souligne ici l'importance de reprendre chaque mot, chaque idée et de ne pas «nettoyer» le témoignage lorsqu'il est répété. L’interprète doit éviter de faire des commentaires personnels, il doit parler à la première personne en utilisant le «je » et, encore une fois, il doit éviter les résumés.

Le troisième élément est relatif à l'impartialité dont l'interprète doit faire preuve. Dans ces circonstances, il est inapproprié qu'une partie au litige, un juge, un parent ou un ami agisse en tant qu'interprète. La Cour mentionne toutefois que des exceptions à ce principe peuvent être possibles lors de procédures préliminaires telle qu'une requête visant la remise en liberté d'un individu qui se déroule en région éloignée. Il serait, en effet, contraire à d'autres dispositions de la Charte, de garder un individu détenu pour la seule raison qu'aucun interprète n'est disponible à court terme compte tenu de l'éloignement du tribunal. Il semble donc, qu'il n'est pas rare, en région, de voir des secrétaires de juges, par exemple, agir comme interprète judiciaire!

Le quatrième élément repose sur la compétence de l'interprète. La Cour reconnaît qu'il n'existe aucun standard ou aucune norme généralement acceptée quant à cet aspect. L'interprétation devra être d'assez bonne qualité pour que justice soit rendue et paraisse avoir été rendue. Le tribunal devra s'assurer de la compétence de l'interprète qui agit devant lui.

Enfin, le cinquième et dernier élément est la concomitance. La Cour mentionne que l'interprétation et la procédure doivent être concomitants. Il n'est pas ici question de la distinction entre une interprétation «simultanée» et une interprétation «successive». Les deux sont acceptables en autant qu'elles soient concomitantes avec la procédure.

Le droit à l'assistance d'un interprète dans le cadre de procédures judiciaires s'est développé, depuis le début du siècle dernier, un peu comme on construit une autoroute. L'analyse juridique sommaire qui précède ne correspond en fait qu'à la voie principale. Néanmoins, les éléments essentiels à une bonne compréhension de la question sont tous là, ce qui nous permet maintenant de passer à un examen sommaire des problématiques découlant de l'exercice de ce droit à l'interprétation.

De façon générale, certains problèmes ont pu être observés ici et là quant à l'exercice du droit à l'interprétation. Je mentionnerai d'abord les principaux pour ensuite traiter de l'aspect plus montréalais de cette question. 
Un premier type de problème pouvant influencer l'interprétation ou le droit à celle-ci, est lié à des perceptions personnelles ou individuelles que peuvent nourrir les différents intervenants du système judiciaire. Pensons ici aux multiples préjugés racistes qui sont trop souvent véhiculés ou encore à l'idée que l'interprète ne soit utilisé que pour faciliter le mensonge en permettant plus de temps de réflexion pour répondre et enfin, aux différences culturelles qui peuvent être frappantes dans un procès, par exemple, où l'interprète punjabi est musulman alors que l'accusé est sikh! Tous ces exemples peuvent certainement influencer le critère d'impartialité dans la norme canadienne d'interprétation.

Une deuxième série de problèmes pourrait être qualifiée de systémique "préprocédural». L'accusé qui ne parle pas l'une des deux langues officielles et qui obtient les services d'un avocat qui ne parle pas sa langue, peut en être sévèrement affecté. D'une part, l'avocat peut effectuer une mauvaise évaluation du besoin d'interprète pour son client dans le cadre des procédures. En effet, si les deux individus peuvent communiquer ensemble dans un environnement tel qu'un bureau ou une salle de réunion, il peut en aller autrement dans une salle de cour dans le cadre d'un contreinterrogatoire serré. D'autre part, cette lacune dans la communication avocat-client se concrétise également par un fréquent manque de préparation de l'affaire. L'avocat discute ordinairement avec son client quelques minutes avant l'audience, bénéficiant de l'interprète assigné par la cour, et la direction que prendra l'affaire reposera malheureusement sur cette trop brève conversation. J'ai moi-même constaté trop souvent que des individus avaient plaidé coupable à des infractions criminelles sur les conseils de leur avocat, sans réellement être au courant de la nature et des conséquences du geste qu'ils venaient de poser.

Enfin, une dernière catégorie de problèmes peut, cette fois, être qualifiée de systémique procédural. Ce sont des problèmes qui apparaissent au cours des procédures et qui dans certains cas, peuvent sembler tout à fait évidents. Les principaux sont les suivants :

- Il est quasi impossible, pour les tribunaux, d'évaluer la compétence d'un interprète. La seule véritable façon d'atteindre un tel objectif repose sur le fait qu'un juge, un avocat ou toute autre personne présente lors de l'audience, parle et comprenne la langue traduite par l'interprète.

- Il n'existe aucun système de normes, de standards, d'évaluation ou d'accréditation, généralement reconnu pour les interprètes du milieu judiciaire. Il existe, en Ontario, une forme d'évaluation sommaire des interprètes judiciaires et le ministère de la Justice du Québec est également allé de l'avant en janvier 2001 avec un processus d'accréditation comportant un mode d'évaluation. Il s'agit toutefois d'efforts provinciaux dont les véritables preuves restent à faire et qui, surtout, profiteront essentiellement aux seuls tribunaux relevant du gouvernement provincial.

- L'utilisation d'argots et de dialectes posent plusieurs problèmes de traduction. L'image du cockney qui n'a jamais quitté Londres qui discute avec un "good old boy» de la Louisiane, en dit long. Les deux individus parlent l'anglais mais risquent de ne pas se comprendre à plusieurs égards. Dans un autre ordre d'idée, les mots qui n'existent pas dans une langue présentent également un défi de taille. La notion de culpabilité, par exemple, n'existe pas pour plusieurs groupes autochtones. Le choix de plaider coupable ou non coupable dans notre système de justice criminel devient donc un casse-tête de traduction lorsque l'accusé est un Inuit ou un Cri.

- Enfin, le style et le ton de l'interprète peuvent parfois être décisifs sur la crédibilité d'un témoin. La façon dont s'exprime un individu peut effectivement être cruciale 
quant à sa crédibilité. Un témoignage rempli d'hésitations, d'impolitesses, de détours linguistiques tels que "you know» ou «well» et qui manque d'assurance, pourrait très facilement être écarté par un tribunal. Il est donc essentiel que l'interprète s'exprime de la même façon que le témoin sans y ajouter aucune touche personnelle et sans rien retrancher de l'expression du témoin.

Les tribunaux sur le territoire de Montréal ne font pas exception à la règle et connaissent, à des degrés différents, ces trois types de problèmes en matière d'interprétation. Toutefois, outre les plaintes de la magistrature concernant les résumés de témoignages et, dans une certaine mesure, la compétence des interprètes, il semble que le problème majeur en matière d'interprétation dans les tribunaux siégeant à Montréal se situe dans la disponibilité des interprètes, particulièrement pour la Cour municipale de Montréal.

Un coup d'œil sur les données démographiques de 1996 révèle que près de $90 \%$ des immigrants au Québec étaient installés dans la grande Région métropolitaine de Montréal qui s'étend sur un territoire de plus de $4000 \mathrm{~km}^{2}$ pour une population de 3.3 millions d'individus.

Le territoire sur lequel se disperse cet important bassin de population d'immigrants, comporte plusieurs tribunaux dont trois majeurs où des services d'interprètes sont plus fréquemment requis: le Palais de justice de Montréal (responsabilité provinciale), la Commission de l'immigration (responsabilité fédérale) et la Cour municipale de Montréal (responsabilité municipale).

Sans être volontairement en compétition dans le «marché des interprètes », ces trois tribunaux le sont inévitablement de façon implicite. En effet, ces trois organismes sont physiquement très rapprochés dans le Vieux Montréal et dans le centre de la ville. Ils desservent la même population et, dans certains cas, dans des affaires très similaires, comme par exemple, l'audition de causes criminelles à la Cour municipale et au Palais de justice. Les procédures pour l'octroi de contrats aux interprètes ne sont pas les mêmes, et, plus important encore, les tarifs peuvent varier substantiellement. Il est arrivé fréquemment que des interprètes, présents dans des procédures en avantmidi à la Cour municipale, ne se soient pas présentés pour la suite de l'audition en après-midi parce qu'ils avaient une assignation plus lucrative à la Commission de l'immigration située quelques coins de rue plus loin!

Le bilinguisme de Montréal caractérise et augmente le besoin pour des interprètes. Comme plusieurs accusés ou témoins sont soit anglophones ou francophones, il est donc usuel de rechercher des interprètes qui traduisent d'une langue source vers l'anglais ou vers le français. Cet exercice n'est pas toujours facile. Certaines communautés, compte tenu de leur passé colonial ou de leur histoire en général, utilisent majoritairement soit le français ou l'anglais. Il est presque impossible, par exemple, de trouver des interprètes tamoul-français. La communauté tamoule à Montréal semble utiliser essentiellement l'anglais comme langue seconde.

Enfin, l'usage de certaines langues peut être excessivement rare ce qui complique d'autant plus la recherche d'un interprète pour celles-ci. À la Cour municipale, nous avons mis plusieurs semaines, l'année dernière, à tenter de trouver un interprète pour le dialecte des Açores. L'examen des données de l'Institut de la statistique du Québec concernant la répartition de la population selon la connaissance des langues non officielles, confirme la rareté de certaines langues dans la région de Montréal. Comment trouver rapidement des gens rencontrant les critères d'interprétation 
élaborés dans Tran lorsque seulement 65 personnes connaissent le macédonien, 210 le cri, 290 le pachto ou 325 le malayalam? Le labiale, le lingala, le gujarati ne sont que quelques exemples de langues pour lesquelles un interprète a dû être assigné à la Cour municipale en 1997 et 1998. On ne retrouve pourtant aucun individu connaissant ces langues dans tout le Québec selon les statistiques de l'Institut!

Bref, la conjugaison de ces facteurs spécifiques à Montréal, vient ajouter un problème supplémentaire dans l'exercice du droit à l'interprétation, soit celui de la disponibilité des interprètes.

En terminant, examinons les pistes de solutions qui pourraient être envisagées afin d'éliminer certains de ces problèmes ou, à tout le moins, d'en limiter le plus possible les effets.

Certains ajustements se situent au niveau des intervenants eux-mêmes. Les interprètes, par exemple, doivent intervenir promptement auprès du tribunal lorsqu'ils ne comprennent pas le sens de concepts légaux ou lorsqu'ils constatent que le témoin ou l'accusé semble avoir des problèmes de compréhension. Les juges et les avocats, de leur coté, devraient tenter d'utiliser une terminologie la plus simple possible dans un procès où intervient un interprète. De plus, avant le début des auditions, les avocats devraient, à l'aide d'un interprète, expliquer sommairement le système judiciaire et les procédures lors de rencontres avec les accusés ou les témoins. Il est également important que ces derniers soient prévenus de signaler au tribunal, tout problème qui peut survenir au cours de l'interprétation.

D’autres solutions, plus systémiques, ont déjà été imaginées. Retenons ici les plus importantes :

- Le développement de standards ou de normes ainsi qu'une forme d'accréditation pour les interprètes;

- Le développement de mécanismes de formation continue pour les interprètes en milieu judiciaire;

- La présence de deux interprètes à la cour: le premier pour la traduction des procédures et le second pour la traduction des communications entre l'avocat et son client. Ce dernier pourrait assurer un certain contrôle de la qualité de l'interprétation fournie par l'autre interprète;

- Un contrôle de la qualité de l'interprétation, postérieur à l'audition et effectué par un deuxième interprète. Ce contrôle, pour être utile pour d'éventuelles procédures d'appel, devrait être exercé systématiquement et quotidiennement dans toutes procédures ayant nécessité la présence d'un interprète. L'utilisation de technologies audio-visuelles serait également nécessaire.

Pour ce qui est de la situation à Montréal, le ministère de la Justice du Québec annonçait récemment, la mise en place, le premier janvier 2001, d'un programme d'évaluation et d'accréditation pour les interprètes judiciaires. Un pas important a ainsi été franchi en vue de régler certains problèmes, notamment ceux reliés à la compétence des interprètes. Le problème de disponibilité risque toutefois de subsister dans l'état actuel des choses et même d'empirer avec chaque nouvelle vague d'immigration.

À mon avis, la solution la plus efficace serait la création d'une forme d'ordre professionnel ou d'un organisme national qui verrait à la formation et à l'accréditation des interprètes dans tous les champs de spécialisation et dont une section serait consacrée au milieu judiciaire. Cet organisme établirait un code de déontologie et un tarif dont l'application serait national. De plus, pour être encore plus visionnaire, à 
l'aide d'une technologie adéquate, l'ensemble des besoins d'interprètes devant les tribunaux pourrait transiter de façon électronique par cet organisme ou une division de celui-ci, ce qui permettrait d'assurer une distribution et une prestation de services d'interprétation équitable, uniforme et de qualité partout sur le territoire.

Le droit à l'assistance d'un interprète lors de procédures judiciaires s'est donc considérablement développé depuis les premiers principes établis par Lord Reading au début du siècle dernier en Angleterre. Les garanties à ce chapitre se sont surtout solidifiées au fil des ans mais non sans éliminer tous les problèmes liés à l'exercice de ce droit. Il reste donc plusieurs grands défis à relever. Des défis qui nécessiteront tôt ou tard une plus grande implication technique et financière de nos gouvernements, pour lesquels l'immigration représente un enjeu de taille pour le maintien et la perpétuelle construction de notre société. Il est inévitable, dans une société qui se «multiculturalise» à un rythme aussi important, que l'érection de tous les types de ponts servant à franchir les barrières des langues, devra être favorisée.

\section{RÉFÉRENCES}

Beaudoin, G.-A. et P. E. Mendes (1996) : «Charte canadienne des droits et libertés», $3^{e}$ éd., Wilson et Lafleur, Montréal, p. 746-761.

Gosselin, J. et G. Laporte: «La Charte canadienne des droits et libertés: Les grands énoncés de la Cour Suprême», vol. 2, mis à jour août 2000, Les Éditions Yvon Blais, Montréal, p. VIII /8-1 à $8-9$.

Heller, D. J. (1995) : "Language Bias In The Criminal Justice System”, 37 Crim. L.Q., 344

McLeod, R. M., J. D. Takach, H. F. Morton and M. D. Segal (2001): "The Canadian Charter Of Rights - The Prosecution and Defence of Criminal and Statutory Offences", vol. 2, Carswell, Toronto, p. 22-1 to 22-31.

Steele, G. J. (1992) : “Court Interpreters in Canadian Criminal Law” 34 Crim. L.Q., 218. 